CAHIERS DE

NARRATOLOGIE

\section{Cahiers de Narratologie}

Analyse et théorie narratives

32 | 2017

Récit et argumentation, interactions, lieux et dispositifs sociaux

\title{
Pour un recadrage (méta-)herméneutique de la narratologie contemporaine. Entretien avec Liesbeth Korthals Altes
}

Par Raphaël Baroni

\section{Raphaël Baroni}

\section{OpenEdition}

Journals

Electronic version

URL: http://journals.openedition.org/narratologie/7884

DOI: 10.4000/narratologie.7884

ISSN: 1765-307X

Publisher

LIRCES

\section{Electronic reference}

Raphaël Baroni, « Pour un recadrage (méta-)herméneutique de la narratologie contemporaine. Entretien avec Liesbeth Korthals Altes », Cahiers de Narratologie [Online], 32 | 2017, Online since 21 December 2017, connection on 21 April 2019. URL : http://journals.openedition.org/narratologie/7884 ; DOI : 10.4000/narratologie.7884

This text was automatically generated on 21 April 2019

Article L.111-1 du Code de la propriété intellectuelle. 


\section{Pour un recadrage (méta- )herméneutique de la narratologie contemporaine. Entretien avec Liesbeth Korthals Altes}

Par Raphaël Baroni

Raphaël Baroni

1 Dans son dernier ouvrage Ethos and Narrative Interpretation. The Negotiation of Values in Fiction (2014), qui a obtenu l'année passée le prestigieux Perkins Prize décerné par l'association internationale pour les études narratives (ISSN) pour le meilleur ouvrage en théorie narrative, Liesbeth Korthals Altes se donne deux objectifs ambitieux : d'une part, montrer l'importance de l'image de l'auteur que se construit le lecteur lorsqu'il s'agit d'attribuer un valeur éthique ou esthétique à une fiction littéraire; d'autre part, adjoindre à la narratologie une perspective méta-herméneutique, qui consiste à réfléchir sur la diversité des cadrages interprétatifs confrontés à des œuvres polémiques ou ambiguës, et sur quelques facteurs qui les conditionnent. Ainsi que le résume Anaïs Oléron:

L. Korthals Altes ne se contente pas d'enrichir et de délimiter la notion d'ethos, somme toute aussi vague que répandue (sa plasticité expliquant d'ailleurs probablement sa valeur opératoire). Elle met au jour certains présupposés de la narratologie et développe ainsi toute une réflexion sur la manière dont pourrait évoluer l'analyse théorique des textes narratifs. Travailler sur la notion d'ethos implique en effet de se placer du côté de l'interprétation des textes, ce qui ne va pas sans difficultés. L. Korthals Altes entend s'attaquer à certains angles morts des études narratologiques, en croisant celles-ci avec d'autres modes d'analyse comme l'herméneutique, la phénoménologie, la sociologie ou les théories cognitives. Il est entendu que certaines de ces méthodes entrent en tension avec la démarche globalement descriptive de la narratologie classique. (2015, \$2-3)

2 C'est donc à un véritable recadrage conceptuel de la narratologie contemporaine et à un métissage disciplinaire que cet ouvrage nous invite, tout en engageant un dialogue interculturel inédit entre une théorie du récit d'orientation rhétorique, qui s'inscrit dans 
le sillage de l'étude de l'auteur implicite et la narration non fiable initiée par Wayne C. Booth, et les travaux qui se situent dans le champ de l'analyse de discours francophone, notamment avec les travaux de Dominique Maingueneau, de Ruth Amossy ou de Jérôme Meizoz sur l'ethos et la posture de l'auteur. Une autre originalité, pour un ouvrage qui s'adresse à un public anglo-saxon, tient au corpus, qui ménage une part importante à l'analyse d'auteurs français controversés, à l'instar de Christine Angot et de Michel Houellebecq.

Raphaël Baroni : Pour commencer cet entretien, j'aimerais vous demander ce qui vous a amené à vous intéresser à ces questions d'ethos, et à lier une perspective rhétorique aux théories contemporaines du récit?

4 Liesbeth Korthals Altes: Cela nous fait revenir en arrière! En 1992, je bouclais mon ouvrage Le Salut par la fiction? Sens, valeurs et narrativité dans Le Roi des aulnes de Michel Tournier. J'avais l'ambition de fournir un appareil narratologique suffisamment subtil pour permettre de décrire les effets-valeur d'un roman aussi complexe que celui de Tournier (dans Poétique des valeurs, paru en 2001, Vincent Jouve renvoie de manière détaillée à cet ouvrage). Au bout de ce travail encore très structuraliste d'inspiration, j'achoppai bien entendu sur la flagrante diversité des lectures, défi de taille pour la narratologue que j'étais, qui voulais avec une candeur bien partagée dans la profession décrire la structure narrative d'un texte. J'ai continué à m'intéresser à ce que pouvaient apporter la narratologie et, de manière plus générale, la théorie narrative à l'analyse du jeu des perspectives narratives en régime de fiction, concernant par exemple la fiabilité ou non-fiabilité d'une voix narrative, l'ironie, ou l'ambiguïté d'un classement générique.

5 Le défi était aussi, pour moi, de repenser les conséquences, pour ma compréhension du phénomène littéraire, de mes échanges avec mes collègues proches - sociologues de l'art et de la littérature, chercheurs en sciences cognitives, psychologues étudiant le changement des valeurs dans la société, etc. J'ai dû repenser ma propre position théorique et méthodologique. J'avais l'impression que nous tournions en rond, qu'il fallait tirer les conséquences méthodologiques de la sémiotique, des approches sociologiques (ou sociocritiques) et historiques de la production du sens textuel, et plus spécifiquement, littéraire; qu'il fallait - de nombreux autres l'ont signalé - inclure dans les modèles narratologiques la diversité des manières de construire cet «objet » littéraire que nous voulons « décrire » et analyser. Il ne s'agissait pas de souligner l'idée que toute lecture est subjective, mais, dans une perspective herméneutique qui réfléchit sur elle-même, de reconstruire des mécanismes cognitifs sous-jacents et les effets de conventions de lecture et autres conceptions normatives, qui expliqueraient cette diversité interprétative. Ce livre sur l'ethos m'a permis de creuser cette question, même s'il suscite probablement plus d'interrogations qu'il ne propose de réponses.

6 En particulier, je me suis demandée comment interagissent dans notre lecture, notre interprétation et notre évaluation d'un texte: (a) l'ethos que nous attribuons à un narrateur, un personnage " porte-valeur ", (b) le genre que nous attribuons à un texte, (c) le type d'auteur auquel nous pensons avoir affaire, et (d) des présupposés et des conceptions normatives qui influencent nos attentes concernant l'expérience littéraire et notre manière de lire et d'évaluer les textes (nos idées sur la littérature, la communication, le Moi...). La notion de cadre, que j'emprunte à Erving Goffman (1974), ou plutôt celle d'acte de cadrage ${ }^{1}$, m'a paru indispensable pour comprendre cette diversité des lectures souvent flagrante, mais que néglige presque totalement la narratologie, qui pourtant se trouve amenée à statuer sur le type de narrateur, sa fiabilité, etc.. Ces actes de 
cadrages, qui interagissent entre eux, sont pour ainsi dire constitutifs, puisqu'ils déterminent la scène énonciative/communicative que le lecteur perçoit comme la plus pertinente. Ils appellent engendrent aussi des horizons d'attente et des régimes de valeurs spécifiques concernant le type d'expérience dans lequel le lecteur pense s'engager ou, rétrospectivement, estime s'être engagé: expérience de portée esthétique, autobiographique, civique, commerciale, médiatique, etc ${ }^{2}$. Dave Eggers (de la génération américaine dite post-ironique) a suscité des conflits d'interprétation incontournables. Ceux-ci, je pense, peuvent être décrits comme des cadrages différents du genre du texte, en fonction de la stratégie et de l'habitude de lecture de l'interprète et de sa perception de la posture et de l'ethos de l'auteur et des voix narratives prépondérantes. Par exemple, pour certains lecteurs - et l'on peut ici se référer à des lectures documentées -, Angot est une écrivaine qui écrit au ras de son réel vécu, avec une sincérité douloureuse, authentique (ethos) ; ces lecteurs ont tendance à lire Sujet Angot comme une autofiction tendant vers l'autobiographique, et parfois même carrément comme un texte autobiographique (cadrage générique). Pour qui considère que la littérature vaut pour sa charge existentielle et expérientielle, ce poids de vécu apparait comme un gage de valeur; pour ceux qui nourrissent une conception disons autonomiste, esthétique, de la littérature, ce parti-pris de réel brut est naï, sinon une imposture, qui met en tout cas en danger la qualité esthétique de l'œuvre (conceptions normatives, régimes de valeurs). Pour un autre groupe de lecteurs, Angot est une auteure typiquement littéraire, pour qui la vie et l'écriture sont des domaines bien distincts, et qui serait donc bien éloignée du spontanéisme affiché par ses personnages et narrateurs perçus comme porte-parole (ethos, posture, scène énonciative). Dans ce cas, on aura tendance à lire Sujet Angot comme une métafiction (genre) qui thématise au lieu de " performer » le rapport entre le vécu et sa mise en forme littéraire, ou qui, peut-être même, exhibe et critique l'exhibition du Moi, impérative dans notre «culture du narcissime » (Christopher Lash) et le voyeurisme avide du public (ethos, genre).

9 L'intensité émotionnelle de ce genre de divergences interprétatives est forte. J'ose affirmer que notre perception de l'ethos de l'auteur joue inévitablement un rôle dans les genres où est en jeu le rapport de l'écriture "littéraire " à une réalité biographique, historique ou sociale: documentaire et docufiction, genres autobiographiques et autofiction, roman historique, roman-essai, roman engagé... Dans ce genre de conflits interprétatifs, il en va non seulement de l'adhésion à des visions du monde, des valeurs et des positions éthiques perçues comme proposées dans l'expérience de lecture, mais aussi des stratégies interprétatives elles-même: quelle manière de lire - réaliste/mimétique, factuelle, psychologique, morale, esthétique - est-elle estimée adéquate? Quel contrat communicationnel et éthique le lecteur estime-t-il être en place ? Il est frappant de constater à quel point les réactions des lecteurs sont véhémentes et se placent sur le terrain de la morale quand un cadrage générique ou un cadrage d'ethos ou de ton s'avèrent non pas seulement ambigus mais faux, comme le montrent les cas de Wilkomirski ou de James Frey. On est loin d'une situation où les distinctions génériques ne compteraient plus, comme il est parfois dit de notre condition culturelle postmoderne.

RB : Pourriez-vous résumer cette démarche que vous définissez comme une approche « méta-herméneutique »?

Cahiers de Narratologie, 32 | 2017 
11 Je voulais d'essayer de comprendre comment l'analyse et l'interprétation d'un texte, littéraire ou autre, se laissent reconstruire comme un emboîtement particulier de cadrages (genre, posture, ethos, scène communicationnelle, etc.) et comme la mise en œuvre d'une attitude interprétative. Le terme rébarbatif et un peu tautologique de métaherméneutique désigne ce travail de reflexion portant non pas tant sur le sens du texte, mais sur les trajectoires interprétatives, sur la façon dont elles prédisposent à percevoir les positions de valeur qu'articulerait le texte et à les juger selon un (ou plusieurs) régime (s) de valeurs spécifique(s). Evidemment, ce genre d'analyse ne tient pas compte de l'impact de facteurs vraiment personnels dans l'interprétation des textes, mais cherche à mettre en relief des conventions interprétatives assez largement partagées. littéraire) suscite un travail sur les valeurs? Elle peut occasionner ce travail (et je m'excuse de la maladresse de la formulation qui suit) à travers un jeu complexe, emboîté, de perspectives sur des perspectives sur des expériences valorisées: tout d'abord, quand nous lisons, nous sommes amenés à imaginer un monde à travers une représentation ellemême perspectivée (et chargée d'évaluations) de personnages qui agissent, perçoivent et réfléchissent dans un monde fictionnel où se laissent également reconstruire des perspectives évaluatives diverses. En outre, notre investissement affectif, intellectuel et moral dans la lecture nous amène à confronter ce monde évoqué et ses jeux de perspective avec notre propre expérience et nos propres valeurs. Tout ceci nous engage dans une gymnastique ou un calcul axiologique incroyablement complexe, qui requiert une série non close de cadrages et de recadrages, toujours à la merci de nouvelles contextualisations qui pourraient donner encore un tour au caléidoscope.

14 Enfin, et cet aspect est trop rarement souligné, dans le cas où nous formulons une interprétation explicite, a fortiori quand nous discutons de notre expérience de lecture avec d'autres, nous ajoutons encore un niveau à cette réflexivité qui porte finalement sur l'expérience humaine, sur comment la comprendre, l'investir de valeur, mais qui porte aussi sur ces procédures d'attribution de sens et de valeurs elles-mêmes. Vu ainsi, l'échange portant sur l'interprétation littéraire offre un riche terrain d'analyse pour qui s'intéresse à la culture comme "distribution de cognitions ", comme "métacognition " (Donald 2006) et comme négociation de valeurs. Mais attention, je ne pense pas que nous soyons loin, ici, de la narratologie!

RB : Selon vous, l'idéal objectiviste (pour ne pas dire scientiste) de la narratologie telle que la concevaient ses pères fondateurs, notamment formalistes et structuralistes, doit être dépassée. Mais la théorie du récit ne risque-t-elle pas de perdre son identité en se refondant sur une base herméneutique? Que restera-t-il alors de l'héritage structuraliste, notamment au niveau de sa terminologie et de son outillage descriptif ? Cet outillage conceptuel peut-il être mis au service d'une analyse méta-herméneutique?

16 LKA : Le riche travail qu'ont accompli les narratologues jusqu'à présent se laisse très bien comprendre comme la formulation synthétique et systématisée d'une connaissance «pratique » du domaine textuel - ou, de façon souvent plus limitée, du domaine narratif littéraire. Les concepts et procédés narratifs dégagés par la narratologie orientent de manière heuristique notre attention sur le potentiel expressif et idéologique formidable mais lié à des contextes spécifiques - des formes narratives. Ironiquement, la narratologie

Cahiers de Narratologie, 32 | 2017 
fonde et justifie ainsi un «close reading » auquel les narratologues de la première heure voulaient justement proposer une alternative, mais qui a vite fait de resurgir, sans aucune mauvaise conscience. Ce qui manque trop souvent à nos «théories", c'est d'un côté la prise de conscience de la part d'interprétation et des conceptions normatives de la littérature dans nos théories narratologiques; et de l'autre côté, la prise en compte de la diversité de la perception de ces formes narratives à travers le temps et les espaces culturels et sociaux, comme aussi de la diversité des manières de lire: celle-ci tout simplement existe, ce qu'une théorie narrative ne saurait interdire.

17 Je pense qu'une discussion et une analyse des valeurs que l'on considère centrales dans la lecture littéraire, et de la diversité des modes de lecture sont d'une grande importance et devraient être menées ouvertement dans les institutions éducatives, les groupes de lecture, etc. Se découvrir faisant de l'herméneutique, comme Monsieur Jourdain de la prose, n'a rien de dégradant, même pas pour une narratologue, et ne signifie aucunement que l'on se voue à une subjectivité tous azimuts. N'oublions pas que l'herméneutique telle que l'envisageait Schleiermacher basait la compréhension intuitive des textes (l'idée que l'interprète peut connaître un auteur mieux qu'il en se connaissait lui-même) sur une expertise incroyablement exigeante. La (re)lecture de Schleiermacher et de Dilthey, que je discute avec mes étudiants, m'a fait comprendre que l'intuition - la compréhension, disons, d'âme à âme à travers le texte, cette ambition " humaniste » facilement ironisée à laquelle se trouve souvent réduite l'herméneutique en général - se laisse analyser comme un savoir culturel qui, une fois accumulé, permet des courts-circuits entre cognitions de type divers, un peu comme est compris actuellement en sciences cognitives le mécanisme de la créativité (pardonnez ma formulation de non-expert). Pour Schleiermacher, dans mes mots à moi, plus nos connaissances du domaine spécifique et de la culture interdiscursive dans lequel fonctionne un texte sont étendues, plus nous sommes capables de comparer celui-ci à un répertoire pertinent et de répondre au défi que représente la compréhension du texte. Je ne dis pas que nous devons reprendre à Schleiermacher l'ambition de reconstituer l'intention de l'auteur. Mais il est utile de mieux comprendre ce que représente chez lui la connaissance intuitive. L'interprétation, selon cette approche, n'est pas nécessairement plus subjective que nombre d'analyses narratologiques qui se laissent rapporter à des programmes herméneutiques normatifs implicites (je développe cette observation dans mon livre).

RB : Un point qui m'a particulièrement frappé, pour un ouvrage rédigé en anglais et publié dans l'une des plus importantes collections américaines dédiées à la théorie du récit («Frontiers of Narrative » aux presses de l'Université du Nebraska), c'est le dialogue que vous engagez entre des perspectives théoriques assez classiques pour les anglo-saxons - notamment la théorie de l'auteur implicite et celle du narrateur non-fiable - et des approches qui sont encore mal connues en dehors de la francophonie, à savoir la théorie de la "posture" telle que l'articulent Jérôme Meizoz ou Alain Viala, et l'analyse du discours littéraire telle que la pratiquent Dominique Maingueneau ou Ruth Amossy. Pouvez-vous nous dire comment ce rapprochement a été rendu possible et de quelle manière il a été reçu par le public anglo-saxon?

19 LKA : J'ai hésité si je devais écrire ce livre en français ou en anglais. Dans les deux cas, il y avait un rôle de médiateur à jouer. Finalement, je me suis dit que le nombre de (jeunes) chercheurs français qui lisent l'anglais est sans doute plus substantiel que celui des anglophones lisant le français et l'allemand. Du coup, j'ai écrit le livre en anglais. Dans la 
recherche française, il y a des orientations particulièrement riches et inspirantes ; John Pier, Sylvie Patron et d'autres ont bien attiré l'attention là-dessus. L'analyse de l'énonciation, la sociocritique - qui ne s'appelle plus ainsi-, l'analyse des valeurs, sont des produits d'exportation française qui méritent diffusion. Cette tradition française combine dans beaucoup de cas des perspectives diverses: historique, linguistique, textuelle, sémiotique et sociologique (ou du moins sociologisante), avec en sus un intérêt passionné pour la littérature et une belle rigueur argumentative. Des noms? Sans souci d'exhaustivité: Bourdieu, bien sûr, mais aussi Philippe Hamon, Alain Viala, Michel Charles, Gisèle Sapiro, Nathalie Heinich, Jérôme Meizoz, et bien sûr votre travail à vous ${ }^{3}$.

Outre-Manche ou outre-Atlantique, je n'ai pas encore perçu une très grande avidité pour une analyse plus systématique des "conditions du sens ». Peut-être cette perspective disons constructiviste est perçue comme remettant en question le primat de l'intérêt existentiel et moral pour la littérature, encore très fort dans les études littéraires en pays anglophones. Ceci dit, je pense tout de suite à des exceptions : Fictions of Authority, de Susan Suleiman (paru en 1992), est un bel exemple combinant une approche rhétorique, sociologique, "gender" et historique, dans une perspective très analytique, où les concepts de posture d'auteur ou d'ethos se dessinent en filigrane.

Ironiquement, l'intérêt éthique de ce regard méta-herméneutique sur les conditions de l'interprétation et de l'évaluation se laisse défendre aussi bien sur un plan théorique (mieux comprendre comment nous en arrivons à des jugements moraux sur des textes fictionnels, et comment ceux-ci en appellent à nos différents systèmes de valeurs), que sur le plan pratique, dans des contextes éducatifs ou professionnels. Il m'est arrivé, ainsi, de travailler avec des petits groupes de lecteurs - adultes en situation professionnelle ou informelle, lycéens - sur un texte littéraire dans le cadre d'un " Atelier d'auto-réflexion ». Nous discutons alors du sens du texte, bien sûr, pour ensuite réfléchir aux différentes stratégies de lecture déployées par les participants, dégageant la manière dont nous cadrons et captons le sens du texte, et les régimes de valeurs souvent divergents auxquels nous nous référons. Tout cela en vue de contribuer à une prise de conscience du même et $d u$ différent, de notre négociation de ce qui représente pour nous ce qui a de la valeur, mais aussi de ce qui caractérise notre propre manière de faire sens, excluant d'autres stratégies et perspectives. Le fait d'avoir à mettre en mots sa propre compréhension du texte, à écouter celle des autres, à les soumettre à une réflexion plus poussée : tout cela constitue un entraînement des facultés cognitives émotionnelles, intellectuelles et morales (éthique de la communication) qui reproduit à un niveau interactif et réfléchi la négociation de positions de valeurs et de perspectives que nous accomplissons déjà durant la lecture, selon des degrés évidemment divers.

RB : Pour revenir à la situation particulière de la narratologie francophone, John Pier a expliqué récemment que « les recherches théoriques sur le récit ont continué en France, mais pas toujours sous le label de la narratologie, dans certains cas, dans des champs non littéraires. L'analyse du discours française semble offrir un cadre conceptuel et méthodologique qui permet de répondre aux questions que se pose la narratologie postclassique " (Pier 2011 : 336). Il est intéressant de noter que les concepts d'ethos et de posture sur lesquelles vous vous appuyez relèvent de la sociologie littéraire ou de l'analyse du discours, alors que vous montrez qu'ils ont une opérativité évidente pour la narratologie contemporaine. Comment expliquezvous cette différence dans les ancrages disciplinaires? Pensez-vous que la narratologie doive être décloisonnée pour intégrer des approches plus larges, 
laissant davantage de place à l'analyse du contexte et des procédures interprétatives ? Est-ce que cette différence de positionnement explique en partie, au-delà de la frontière de la langue, l'absence de dialogue entre les théories de l'auteur implicite et les concepts d'ethos et de posture?

LKA : Personnellement, je suis convaincue de l'importance du dialogue interdisciplinaire. Dans la constitution d'une discipline, on le sait, entrent beaucoup de hasard et de facteurs institutionnels. Les angles morts ne se perçoivent souvent que dans cette confrontation avec des manières différentes de théoriser les mêmes phénomènes. Il me semble que du côté français, comme je l'ai dit plus haut, la recherche sur la forme littéraire, narrative, ou stylistique, et la recherche en sociologie ou sociocritique de l'art et de la littérature ont beaucoup à nous apporter, d'autant plus si nous réussissons à les intégrer, comme vous en montrez vous-même l'exemple (Baroni 2009 ; 2014).

RB : Pouvez-vous encore nous dire quelques mots sur la place importante que tient l'analyse de l'œuvre de Michel Houellebecq dans votre ouvrage ? Peut-on considérer que ce dernier joue un rôle de révélateur sur la place centrale que tient l'auteur dans l'interprétation et dans l'évaluation des valeurs éthiques et esthétiques qui s'attachent à une œuvre?

LKA: L'œuvre de Houellebecq est presque incontournable quand on veut montrer la nécessité de prendre en compte l'auteur dans nos théories sur la circulation sociale de la littérature, vu sa présence (ou son absence, qui elle aussi a fait beaucoup de bruit!) dans les média. Vous le montrez bien vous-même (Baroni 2014), comme le fait aussi Jérôme Meizoz (2007). C'est d'abord la critique littéraire en moi qui s'est sentie interpellée par cette œuvre difficile à cadrer. Depuis, mon intérêt est devenu davantage métaherméneutique. Samuel Estier me l'a d'ailleurs reproché, dans son excellent petit livre sur le style de Houellebecq (Estier 2015). On peut porter différentes casquettes, et je tâcherai d'assumer plus clairement mes responsabilités civiques dans un prochain article!

RB : Pensez-vous que la question de l'éthos se pose également au-delà du champ littéraire, pour des œuvres filmiques ou des récits en bandes dessinées ? Y a-t-il une spécificité des récits verbaux concernant cette question de la voix auctoriale?

LKA : Je pense que la question de l'ethos se pose pour toute communication, et pour toute attribution de sens. Nous calculons, serait-ce de manière inconsciente, le degré de fiabilité, d'autorité, etc. d'un énoncé et d'une énonciation. Des effets de caractère, d'autorité ou de fiabilité déterminent et modalisent comment nous comprenons ce que nous percevons comme un signe, qu'il soit oral, écrit, visuel, ou performé par un corps. Les politiciens le savent très bien ou l'apprennent à leurs dépens, tout comme les banquiers, les chercheurs ou les amoureux. Pour en rester à l'art: je gage que la supputation de l'ethos de l'auteur/de l'artiste joue un rôle décisif dans tous les cas d'art controversé, même si l'artiste est inconnu, ou s'il s'agit d'un collectif. Un cas spécial se présente quand l'œuvre est produite par un agent non-humain, par exemple le chimpanzé Pierre Brassau ou un ordinateur ; ce genre de cas - souvent monté en attrapenigaud - montre justement, par le désarroi interprétatif et évaluatif qu'il suscite, l'importance de l'attribution d'intentions et d'un ethos dans notre engagement dans l'expérience esthétique.

28 Avec mes étudiants nous avions travaillé sur la réception - et les cadrages - de Piss Christ, une œuvre de l'artiste américain Serrano, exposée il y a quelques années au musée d'art moderne de Groningue, aux Pays-Bas. Il s'agit d'un crucifix plongé dans une bassine 
remplie de l'urine de l'artiste. Cette œuvre, perçue par de nombreux critiques et publics comme blasphématoire, a suscité un tollé aux Etats-Unis. Or, parmi les nombreuses réactions, on trouve sur internet une défense de l'œuvre par une critique d'art, ellemême religieuse, sœur Wendy Beckett, qui «redressait » l'ethos attribué à Serrano en comparant son geste, apparemment humiliant et désacralisant, à la tradition catholique du Christ aux outrages. Comme quoi, beaucoup dépend du cadrage...

Quant à l'autre question, s'il y a une spécificité des récits verbaux concernant cette question de la voix auctoriale : je suis tentée de répondre oui et non. Oui, dans la mesure évidemment où le matériau dont est faite l'œuvre littéraire, c'est du langage : celui-ci demeure, malgré toute l'importance du visuel (ou plus généralement, du sensoriel), le medium de la réflexion articulée et d'une vision du monde. L'art verbal interpelle plus évidemment notre idéologie, notre morale, nos manières de conceptualiser notre vision du monde. Mais l'attribution d'un ethos à un énonciateur repose sur une médiation complexe, qui inclut beaucoup d'éléments non-discursifs, non-verbaux, et qui débordent la lecture proprement dite de l'œuvre. Cela inclut les images d'un auteur dans les médias, les effets de sa présence physique lors de séances de lecture, son ethos préalable, etc.. L'ethos que nous nous construisons pour un auteur, ou n'importe quelle instance que nous pensons douée d'intentionalité, se nourrit de bric et de broc. Il est fort possible que l'expressivité d'un corps, l'effet violoncelle d'une voix de basse (je ne parle pas de Houellebecq!) ou d'une manière de parler qui semble proche du corps ou de l'émotion, supplante en efficacité le contenu argumentatif de l'énoncé, qui, lui, constitue l'appel au logos, pour employer les termes d'Aristote. Il suffit de penser au succès de Trump et de comparer les composantes de son ethos discursif et non-discursif à celui d'Obama.

RB : Pour conclure, comment définiriez-vous l'évolution de la théorie du récit contemporaine, dans le monde francophone et au-delà ? Quelles sont les pistes les plus prometteuses pour les études littéraires?

LKA : Ma réponse est liée aux thèmes que, pour ma part, je considère importants et qui tiennent à ma vision de monde et à ma compréhension du rôle que pourrait jouer la littérature dans une démocratie. Côté recherche, comme je l'ai dit plus haut, il me semble que l'ouverture des approches narratologiques ou stylistiques aux dimensions sociologiques, historiques et cognitives est importante. Elle nous permet de bien tenir compte de la construction du sens comme phénomène à la fois social et individuel. La coopération avec les sciences sociales et cognitives offre des possibilités passionnantes, ainsi que le montrent les recherches sur l'exercice des fonctions cognitives - empathie, mind reading, perspective-taking - que peut stimuler la lecture sous certaines conditions; celles aussi qui portent sur notre capacité d'internaliser des valeurs divergentes de celles qu'on pense posséder, ou sur les conditions d'un changement de valeurs; celles enfin qui s'intéressent à l'importance, pour une société, d'une éducation qui stimule l'imagination, la créativité langagière, mais aussi l'articulation de la pensée, l'argumentation et ce que Habermas appelle une éthique du discours, dans des échanges dirigés autour de l'interprétation d'œuvres littéraires ou artistiques (versant appliqué, évidemment normatif). Toutes ces questions sont à explorer, et avec quelque urgence, vu le statut pas toujours florissant de la littérature - mais aussi de l'interprétation raisonnée et de la culture langagière, notamment argumentative - dans l'enseignement ou dans la sphère publique. Avec un œil sur l'institutionnel, je pense que nous avons intérêt à joindre nos forces avec les études cognitives (psychologiques, anthropologiques et autres) pour essayer de mieux expliquer à un public non-narratologue ou non-littéraire l'importance 
social, moral et culturel des divers « usages » de la fiction (voir par exemple Citton 2010, Felski 2008, Collins 2010). Sans crainte de perdre de vue l'importance de la dimension esthétique. Mais celle-ci est également à repenser.

\section{BIBLIOGRAPHY}

Amossy, Ruth. Images de soi dans le discours. La Construction de l'ethos. Lausanne \& Paris : Delachaux et Niestlé, 1999.

Baroni, Raphaël. L'CEuvre du temps. Poétique de la discordance narrative. Paris : Seuil, 2009.

Baroni, Raphaël. « La guerre des voix : critique polyphonique et divergences interprétatives dans l'œuvre de Michel Houellebecq. » COnTEXTE, Varia.

En ligne, page consulté le 23 janvier 2017, URL : http://contextes.revues.org/5979

Baroni, Raphaël \& Francis Langevin (dir.). « Polyphonies : voix et valeurs du discours littéraire », Arborescence, $n^{\circ} 6$, 2016. En ligne, page consultée le 23 janvier 2017, URL : http://www.erudit.org/ revue/arbo/2016/v/n6/index.html

Berger, Peter Ludwig \& Thomas Luckmann. The Social Construction of Reality: A Treatise in the Sociology of Knowledge. Harmondsworth : Penguin Books, 1991 [1966].

Boltanski, Luc \& Laurent Thévenot. De la justification. Les économies de la grandeur. Paris : Gallimard, 1991.

Citton, Yves. L'Avenir des humanités. Économie de la connaissance ou cultures de l'interprétation? Paris : La Découverte, 2010.

Collins, Jim. Bring on the Books for Everybody: How Literary Culture Became Popular Culture. Durham : Duke University Press, 2010.

Donald, Merlin. « Arts and the Cognitive Revolution. » In The Artful Mind : Cognitive Science and the Riddle of Human Creativity. Mark Turner (dir.), New York \& Oxford : Oxford University Press, 2006 p. 3-20.

Estier, Samuel. A propos du «style» de Houellebecq. Retour sur une controverse (1998-2010). Lausanne : Archipel/Essais, 2015.

Felski, Rita. The Uses of Literature. Oxford : Blackwell, 2008.

Goffman, Erving. Frame Analysis : An Essay on the Organization of Experience. New York : Harper \& Row, 1974.

Habermas, Jürgen. Moralbewusstsein und kommunikatives Handeln. Frankfurt am Main : Suhrkamp Taschenbuch, 1983.

Jouve, Vincent. Poétique des valeurs. Paris : Presses Universitaires de France, 2001.

Korthals Altes, Liesbeth. Le Salut par la fiction? Sens, valeurs et narrativité dans le Roi des aulnes de Michel Tournier. Amsterdam \& Atlanta : Rodopi, 1992.

Korthals Altes, Liesbeth. Ethos and Narrative Interpretation. The Negotiation of Values in Fiction, Lincoln \& Londres : University of Nebraska Press, 2014. 
Korthals Altes, Liesbeth. « Actes de cadrage, narratologie et herméneutique - À propos de l'indétermination énonciative dans Sujet Angot de Christine Angot ». Arborescence, n 6, 2016, p. 94-120. En ligne, page consultée le 23 janvier 2017, URL : http://www.erudit.org/revue/ arbo/2016/v/n6/1037506ar.pdf

Meizoz, Jérôme. Postures littéraires. Mises en scène modernes de l'auteur. Genève : Slatkine, 2007. Oleron, Anaïs. « La méta-herméneutique, ou l'analyse des pistes interprétatives. » Acta fabula, vol. 16, $\mathrm{n}^{\circ}$ 1, 2015. En ligne, page consultée le 15 juillet 2015, URL : http://www.fabula.org/acta/ document9099.php

Pier, John. « Is There a French Postclassical Narratology? » In Current Trends in Narratology, Greta Olson (dir.), Berlin \& New York : De Gruyter, 2011, p. 336-367.

Schleiermacher, Friedrich. « The Hermeneutics : Outline of the 1819 Lectures. » New Literary History, vol. 10, $\mathrm{n}^{\circ} 1,1978$, p. 1-16.

Suleiman, Susan. Authoritarian Fictions : the Ideological Novel as a Literary Genre. New York : Columbia University Press, 1983.

\section{NOTES}

1. Sur cette notion, voir aussi l'article de Liesbeth Kothals Altes publié en français dans la revue Arborescence (2016).

2. Sur la notion de régimes de valeurs, voir Boltanski et Thévenot (1991).

3. Voir par exemple Baroni \& Langevin (2016).

\section{AUTHOR}

\section{RAPHAËL BARONI}

Université de Lausanne 